\title{
Usefulness of The Reflux Symptom Index in the Management of Laryngopharyngeal Reflux
}

\author{
https://doi.org/10.47210/bjohns.2020.v28i3.294
}

Saai Ram Thejas, ${ }^{1}$ Ganganamoni Rajamohan, ${ }^{1}$ Sindu Mohan, ${ }^{2}$ Kowsalya Swarna ${ }^{2}$

\section{$\underline{\text { Introduction }}$}

\section{ABSTRACT}

Laryngopharyngeal Reflux (LPR) is highly prevalent in the general population and its impact on health systems is growing dramatically by the day. The contents of the stomach flowing back into the oesophagus, pharynx and larynx because of a transient relaxation of the lower oesophageal sphincter leads to a spectrum of symptoms diagnosed as LPR and Gastroesophageal Reflux Disease (GERD). The aim was to study in detail the symptoms of LPR and to ascertain if and how they hamper the routine of an individual by using the Reflux Symptom Index (RSI).

\section{Materials and Methods}

The Reflux Symptom Index (RSI) is a self-administered nine-item outcomes instrument for LPR. Ninety-one patients with clinically diagnosed LPR were taken up for this study and were issued the RSI (translated into the local language for better results) before and after treatment. Data was assessed at the end of 3 months and 6 months.

\section{$\underline{\text { Results }}$}

The tabulated data showed significant improvement in the symptomatic index after treatment.

Conclusion

It can thus be concluded that RSI is still highly valid in the follow-up for patients with LPR because it can be easily administered and gives accurate results with excellent validation.

$\underline{\text { Keywords }}$

Laryngopharyngeal Reflux

$\mathrm{R}$ eflux of gastroduodenal contents into the larynx because of transient relaxation of the upper and lower oesophageal sphincter may cause inflammation and symptoms resulting in chronic laryngeal signs often referred to as Laryngopharyngeal Reflux (LPR). ${ }^{1}$ It is often associated with chronic cough, hoarseness, dysphonia, recurrent throat clearing and

1 - Department of Otorhinolaryngology, RVM Institute of Medical Sciences, Mulugu Mandal, Telangana

2 - Department of Otorhinolaryngology, Meenakshi Medical College,Hospital \& Research Institute, Kanchipuram, Tamil Nadu

\section{Corresponding author:}

Dr Saai Ram Thejas

email: 21thejas@gmail.com globus pharyngeus (sensation of a lump in the throat).

Due to nonspecific symptoms, laryngoscopy is often performed to rule out malignancy and the diagnosis of LPR is considered in the presence of any signs of laryngeal inflammation. ${ }^{2}$ The two predominant pathophysiological mechanisms for LPR are direct and indirect exposure of the larynx to injurious gastric contents. The direct exposure is due to exposure of laryngopharyngeal mucosa to acid, pepsin and bile acid. The indirect mechanism is thought to be a result of refluxate interactions with structures distal to the larynx, evoking a vagus nerve mediated bronchoconstriction. ${ }^{3}$ LPR is estimated to account for $10 \%$ of all ear, nose and throat clinic patients and $50 \%$ of all patients with voice complaints. ${ }^{4}$ However, due to the lack of a gold-standard in testing, the prevalence of LPR can be overstated, with 
one meta-analysis that reviewed data from $\mathrm{pH}$ probe readings reporting that $10 \%$ to $60 \%$ of normal subjects demonstrated reflux. ${ }^{5}$

LPR differs from classic Gastroesophageal Reflux Disease (GERD) in many ways. While heartburn and regurgitation are common symptoms of GERD, such symptoms are not present in most LPR patients. ${ }^{4}$ LPR, on the other hand presents with laryngeal inflammation and reflux in upright position. LPR is the preferred term for use in Otorhinolaryngology. It is also ubiquitous and pernicious in paediatric patients. The diagnosis may be particularly difficult to make because infants and children almost never complain of heartburn or other symptoms related to reflux. Physiologic barriers against the development of reflux include the lower oesophageal sphincter, oesophageal peristalsis, saliva that buffers the refluxed contents and mucus covering of the stomach-oesophagus-larynx-pharynx along with the upper oesophageal sphincter.

Shaw et al presented a 12-item symptom questionnaire to assess GERD, but again it concentrates on symptoms such as acid taste, burning and chest pain. ${ }^{6}$ The questionnaire from a GERD perspective and from a LPR perspective has to be different since the symptoms vary ever so slightly. The LPR questionnaire must focus more on the symptoms relating to throat and voice as compared to the GERD questionnaire which needs to assess the condition of the oesophagus with relation to the damage done by the anti-peristaltic movement.

Till 2002, there was no validated and approved questionnaire for the diagnosis and follow-up of patients with LPR. The Reflux Symptom Index (RSI) is a selfadministered nine-item score sheet for the symptomatic assessment of patients with Laryngopharyngeal Reflux. It was developed by Belafsky et al and it has been in use ever since. ${ }^{7}$ The RSI has its advantages in the fact that it is easy to use, inexpensive and is easily available in print. A copy can be simply administered to the patient. Thus, this criterion-based score has become an important tool in diagnosis.

This study deals with the relevance of the RSI in local population whilst treating properly diagnosed patients with LPR. Diagnosis was made on the basis of RSI and Laryngeal Endoscopy findings. Data was accumulated and analysed at the start of treatment and $3 \& 6$ months thereafter.

\section{Materials and Methods}

This prospective study was conducted between January 2018 and December 2019 over a period of 24 months.

The patients included in the study were diagnosed with Laryngopharyngeal Reflux, had RSI greater than or equal to 13 , showed laryngeal endoscopy findings secondary to reflux, were 18 years and above, gave consent to treatment and procedure and agreed to be part of the study.

Patients with co-morbidities, Gastroesophageal Reflux Disease, extremes of age, severe disease requiring surgical intervention and those found unfit to take treatment for a 6-month period, were excluded from the study.

All statistical analyses were performed using SPSS Statistics 19 for Windows (IBM Corp., Armonk, NY, USA). Samples were compared and evaluated by means of a paired t-test. A p-value of $<0.05$ was considered statistically significant. The confidence interval was set at $95 \%$.

All the 91 patients presented to the Out Patient Department of Otorhinolaryngology with complaints related to difficulty in swallowing, difficulty in speech, sensation of foreign body in the throat, voice changes and a feeling of acid in the mouth. Such patients were assessed with a Laryngoscopy and the Reflux Symptom Index instrument. RSI $>/=13$ was taken as significant. ${ }^{7}$

According to the Reflux Finding Score (RFS), the various laryngeal changes which can be seen in these patients on a laryngeal endoscopy include Oedema (Vocal cords/Subglottis), Ventricular obliteration, Erythema/hyperaemia, Mucus, Granuloma/granulation. ${ }^{8}$

After thorough counselling and acquiring proper consent, each patient was given the RSI instrument before the beginning of the treatment. If the patient was unable to understand English, the same was explained in the local language (Tamil) and a translated version of the same was given (Tables I and II). The risks of treatment and the necessity to continue for a particular amount of time were mentioned. 
Table I: Reflux Symptom Index in English. ${ }^{7}$

\begin{tabular}{|c|c|c|c|c|c|c|}
\hline $\begin{array}{l}\text { WITHIN THE LAST MONTH, HOW DID THE } \\
\text { FOLLOWING PROBLEMS AFFECT YOU? }\end{array}$ & \multicolumn{6}{|c|}{$\begin{array}{c}\text { 0 = NO PROBLEM } \\
5=\text { SEVERE PROBLEM }\end{array}$} \\
\hline 1. Hoarseness or a problem with your voice & $\mathbf{0}$ & 1 & 2 & 3 & 4 & 5 \\
\hline 2. Clearing your throat & $\mathbf{0}$ & 1 & 2 & 3 & 4 & 5 \\
\hline 3. Excess throat mucus or post nasal drip & $\mathbf{0}$ & 1 & 2 & 3 & 4 & 5 \\
\hline 4. Difficulty in swallowing food, liquids or pills & $\mathbf{0}$ & 1 & 2 & 3 & 4 & 5 \\
\hline 5. Coughing after you ate or after lying down & $\mathbf{0}$ & 1 & 2 & 3 & 4 & 5 \\
\hline 6. Breathing difficulties or choking episodes & $\mathbf{0}$ & 1 & 2 & 3 & 4 & 5 \\
\hline 7. Troublesome or annoying cough & $\mathbf{0}$ & 1 & 2 & 3 & 4 & 5 \\
\hline $\begin{array}{l}\text { 8. Sensation of something sticking in your throat or a lump } \\
\text { in your throat }\end{array}$ & $\mathbf{0}$ & 1 & 2 & 3 & 4 & 5 \\
\hline $\begin{array}{l}\text { 9. Heart burn, chest pain, indigestion or stomach acid } \\
\text { coming up }\end{array}$ & 0 & 1 & 2 & 3 & 4 & 5 \\
\hline
\end{tabular}

\begin{tabular}{|c|c|c|c|c|c|c|}
\hline $\begin{array}{lrr}\text { கடந்த ஒரு } & \text { மாதத்தில் , } \\
\text { கஷ்டங் கள் } & \text { கங்கள் } & \text { கள் } \\
\text { பாதித்தது? } & & \text { எப்படி }\end{array}$ & \multirow[t]{2}{*}{$\begin{array}{l}0 \\
4 \\
8 \\
5 \\
8\end{array}$} & \multicolumn{3}{|c|}{$\begin{array}{l}0 \quad \text { = } \\
\text { பிரச்சு த } \\
\text { இல்லைம் } \\
5=\text { கடுமையான } \\
\text { சிக் கல் }\end{array}$} & \multicolumn{2}{|c|}{ எந் த } \\
\hline $\begin{array}{l}\text { 1. கூச்சல் அல்லது உங்கள் குரலில் } \\
\text { சுக் கல் }\end{array}$ & & 1 & 2 & 3 & 4 & 5 \\
\hline $\begin{array}{l}\text { 2. உங் கள் தொண்டையை } \\
\text { செய் து கொள் வது }\end{array}$ & 0 & 1 & 2 & 3 & 4 & 5 \\
\hline $\begin{array}{l}\text { 3. அதிகப்படியான தொண்டை சளி } \\
\text { அல்லது பிந்தைய நாச சொட்டு }\end{array}$ & 0 & 1 & 2 & 3 & 4 & 5 \\
\hline $\begin{array}{l}\text { 4. உணுவுகள, திரவங் கள் அல்லது } \\
\text { மாத்திரைகளை விழு குவதில் } \\
\text { சரரமம் }\end{array}$ & 0 & 1 & 2 & 3 & 4 & 5 \\
\hline $\begin{array}{l}\text { 5. நீங்கள் சாப்பிட்ட பிறகு அல்லது } \\
\text { படுத்த பிறகு இருமல் }\end{array}$ & 0 & 1 & 2 & 3 & 4 & 5 \\
\hline $\begin{array}{l}\text { 6. சுவாசிப்பதில் சிரமங் கள் அல்லது } \\
\text { மூச் சுத் தணணறல் கள் }\end{array}$ & 0 & 1 & 2 & 3 & 4 & 5 \\
\hline $\begin{array}{l}\text { 7. தொந் தரவான் } \\
\text { எரிச்சலூட்டும் இருமல் }\end{array}$ & 0 & 1 & 2 & 3 & 4 & 5 \\
\hline $\begin{array}{l}\text { 8. உங் கள் தொண்டையில் ஏதோ } \\
\text { ஓட்டிக் கொண் டிருக் கும் உண் ர் } \\
\text { அல்லது உங் கள் தொண் டையில் } \\
\text { ஓரு கட்டி }\end{array}$ & 0 & 1 & 2 & 3 & 4 & 5 \\
\hline $\begin{array}{l}\text { 9. நெஞ்செரிச்சல், மார்பு வலி, } \\
\text { அஜீரணும் அல்லது வயிற் று } \\
\text { அமிலம் வரும் உணூர்வு }\end{array}$ & 0 & 1 & 2 & 3 & 4 & 5 \\
\hline
\end{tabular}

Table II: Reflux Symptom Index in Tamil 


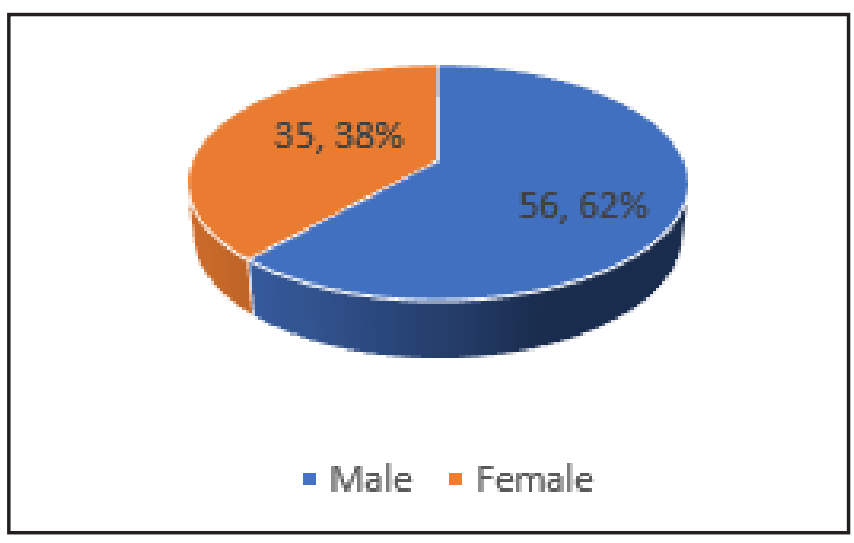

Fig. 1. Gender Distribution

The RSI instrument contains 9 questions. Each question carries 5 points on a $0-5$ severity scale wherein 0 indicates no problem and 5 indicates severe problem. Each patient was asked to fill this up without any prompting. Thus, the maximum score a patient could get was 45 . Any value of $>/=13$ was considered significant and part of study. ${ }^{7}$

The treatment modality was multi-fold and each patient was asked to follow the same pattern.

The dietary modifications included no eating or drinking within 3 hours of bedtime, avoiding over eating or reclining right after meals, fried food, coffee, tea, chocolate, mints, soda, alcohol, sticking to low fat diet and a particular increase in the intake of water.

The lifestyle modifications included elevation of the head end of the bed, avoiding wearing tight fitting clothes or belts and quitting tobacco.

The Medical management included Liquid Antacid - Magnesium Hydroxide and Aluminium Hydroxide (1 tablespoon after each meal and at bedtime) and Proton Pump Inhibitor (PPI) - Pantoprazole, $20 \mathrm{mg}$ half an hour before the first and last meals of the day.

After the aforementioned therapy, patients were asked to follow-up once a month for the next 6 months. The RSI instrument was again given to the patient at the end of 3 months and 6 months. The results were tabulated. After the end of the 6-month period, patients were asked to slowly wean off the medications and strictly follow the lifestyle changes to keep the symptoms under control.

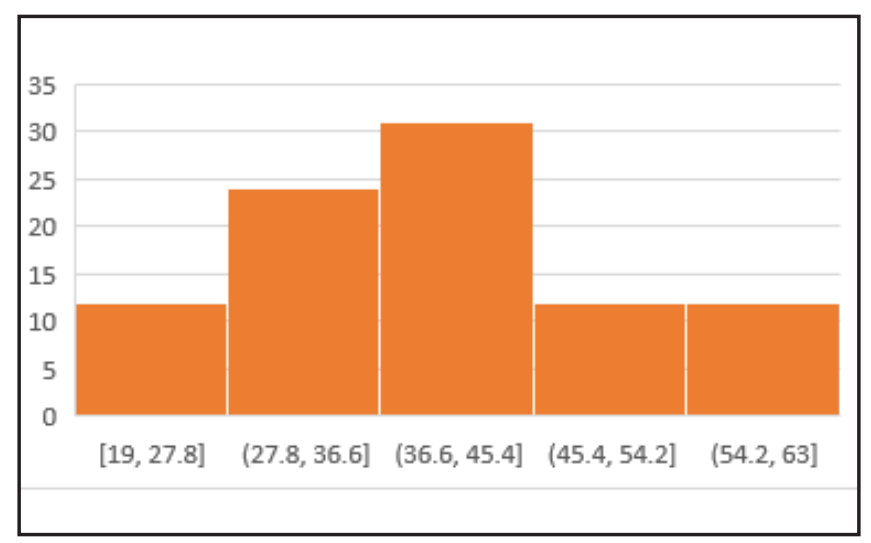

Fig.2. Age Distribution

\section{Results}

Among the 91 people included in the study, 56 were male and 35 were female. (Fig. 1)

The average age of the study group was found to be 39.62 years. The maximum number of patients were found to be in the age bracket of 36-45 years. The youngest case reported in our study was 19 years with the oldest being 62 years. (Figure 2)

The average RSI score before treatment was 23.78. Before treatment, the symptom causing the most trouble was "Heart burn, chest pain, indigestion or stomach acid coming up" which docked at 4.109/5 and the symptom causing the least trouble was "Breathing difficulties or choking episodes" which docked at 1.637/5.

At the end of 3 months, the sensation of heart burn/ acid in throat improved by about $56 \%$ and by the end of 6 months it reached to about $65 \%$.During the treatment i.e. at the 3-month and 6-month intervals, the relative improvement was found to be the most in "Difficulty in swallowing food, liquids or pills" at a significant $11 \%$.

The total score improved by about $49 \%$ after 3 months and $58 \%$ after 6 months (Table III) and the p-value was found to be significant (Tables IV \& V).

\section{Discussion}

There is a difference in the symptomatology of GERD and LPR. The patterns, mechanisms, manifestations and treatment of LPR and GERD differ and the 
Table III: Average RSI Score at 0, 3 and 6 month timeline

\begin{tabular}{|l|c|c|c|}
\hline $\begin{array}{c}\text { WITHIN THE LAST MONTH, HOW DID THE } \\
\text { FOLLOWING PROBLEMS AFFECT YOU? }\end{array}$ & BEFORE & $\begin{array}{c}\text { AFTER 3 } \\
\text { MONTHS }\end{array}$ & $\begin{array}{c}\text { AFTER 6 } \\
\text { MONTHS }\end{array}$ \\
\hline Hoarseness or a problem with your voice & 2.538 & 1.318 & 1.055 \\
\hline Clearing your throat & 2.142 & 1.142 & 0.912 \\
\hline Excess throat mucus or post nasal drip & 2.406 & 1.241 & 1.165 \\
\hline Difficulty in swallowing food, liquids or pills & 3.032 & 1.571 & 1.253 \\
\hline Coughing after you ate or after lying down & 1.912 & 1.021 & 0.868 \\
\hline Breathing difficulties or choking episodes & 1.637 & 0.978 & 0.868 \\
\hline Troublesome or annoying cough & 2.56 & 1.274 & 1.066 \\
\hline $\begin{array}{l}\text { Sensation of something sticking in your throat or a lump in } \\
\text { your throat }\end{array}$ & 3.439 & 1.67 & 1.429 \\
\hline $\begin{array}{l}\text { Heart burn, chest pain, indigestion or stomach acid coming } \\
\text { up }\end{array}$ & 4.109 & 1.824 & 1.418 \\
\hline TOTAL & 23.775 & 12.044 & 10.034 \\
\hline
\end{tabular}

gastroenterology model of reflux disease does not apply to LPR. LPR patients have head and neck symptoms, but heartburn is uncommon. Consequently, LPR is often called silent reflux. LPR patients have predominantly upright (day-time) reflux and normal oesophageal motility; most do not have oesophagitis, which is the diagnostic entity of GERD. Laryngopharyngeal epithelium is far more susceptible to reflux-related tissue injury than is the oesophageal epithelium. Because of these differences, treatment algorithms for LPR and GERD vary. ${ }^{9}$
The RSI is a nine-item self-administered outcome instrument that accurately documents symptom improvement of patients with LPR, thus displaying excellent criterion-based validity.?

The questionnaire from a GERD perspective and from an LPR perspective has to be different since the symptomatology vary ever so slightly. The LPR questionnaire must focus more on the symptoms in the throat and voice as compared to the GERD questionnaire which needs to assess the condition of the oesophagus with relation to the damage done by the anti-peristaltic

Table IV: Paired Sample Correlations after 3 months.

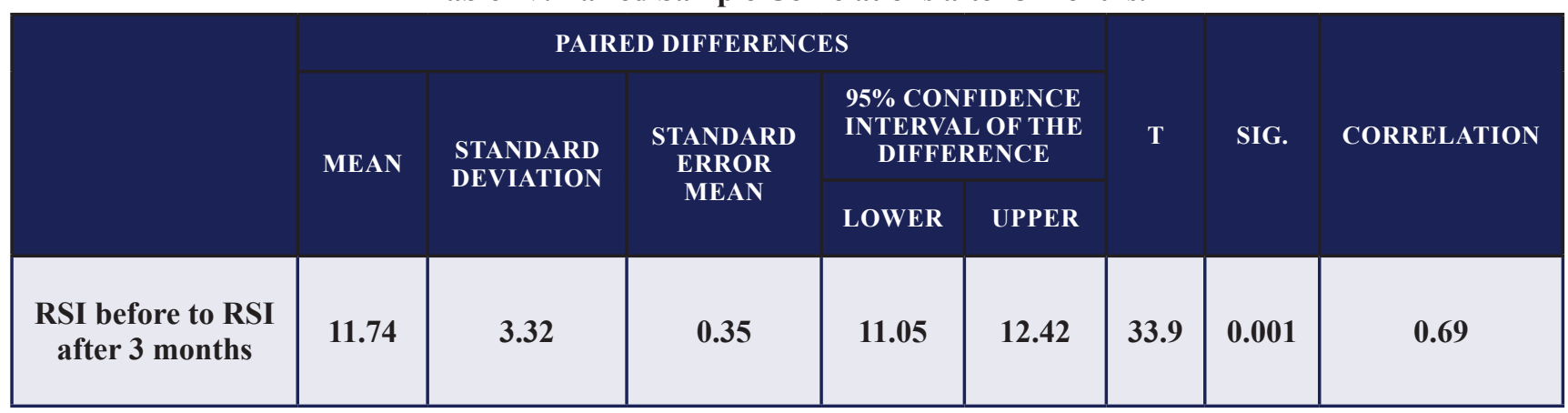

SD: Standard deviation, CI: Confidence interval, SE: Standard error 
Table V: Paired Sample Correlations after 6 months.

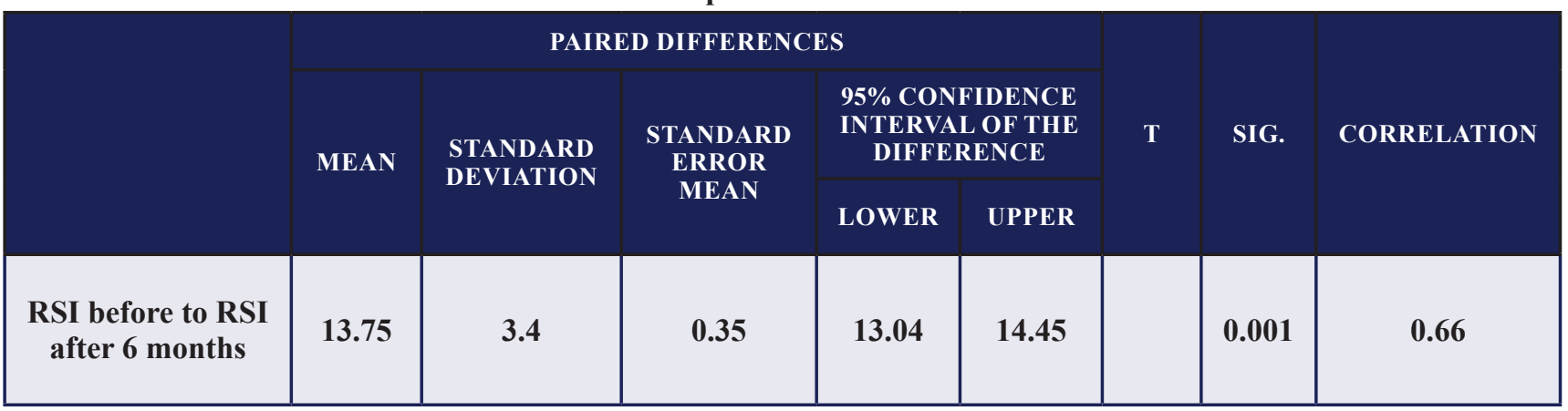

SD: Standard deviation, CI: Confidence interval, SE: Standard error

movement.

Symptoms of LPR improve over 2 months of therapy. The physical findings of LPR resolve more slowly than the symptoms and this continues throughout at least 6 months of treatment. The physical findings of LPR are not always associated with patient symptoms and treatment should continue for a minimum of 6 months or until complete resolution of the physical findings. ${ }^{10}$

The diagnosis of LPR is usually made on the basis of presenting symptoms and associated laryngeal signs. Current recommendation for management of this group of patients is empiric therapy with twice daily protonpump inhibitors for 2-4 months. ${ }^{11}$ In majority of those who are unresponsive to such therapy, other causes of laryngeal irritation is considered.

Although acid can be controlled by proton pump inhibitor (PPI) therapy, all of the other damaging factors (i.e. pepsin, bile salts, bacteria and pancreatic proteolytic enzymes) remain unchanged on PPI therapy and may have their damaging ability enhanced. ${ }^{12}$

Changing food, lifestyle and habits will go a long way in reducing the drug intake and maintaining long term cure. Laryngopharyngeal reflux still has many aspects in its etio-pathogenesis as well as treatment which need to be understood before we can achieve complete cure rates. ${ }^{13}$ Awareness regarding LPR in general population will also help reduce the severity with which the patients present to the primary care physician.

PPIs remain the cornerstone of treatment of LPR. The current management recommendation for this group of patients is empiric therapy with twice-daily PPIs for 1 to 2 months. ${ }^{14}$

Various studies have found that the RFS as a clinical tool for diagnosis of LPR should be viewed with limited utility as it exhibits low inter-rater reliability. ${ }^{15}$ Thus, it becomes all the more necessary that the RSI be assessed extensively for symptomatic betterment of the patient.

Every fifth patient who reports to their family medicine physician shows symptoms of LPR. At primary health care levels, it is possible to establish some form of prevention, diagnostics and therapy for LPR in accordance with suggested algorithms. Only a small number of patients require surgical procedures and do not show improvement with medical management. ${ }^{16}$

Research has proved that the RSI is a quality tool in the assessment of LPR. It can be used in regular ENT practice to analyse the improvement in patients after the commencement of therapy. ${ }^{17,18}$

\section{Conclusion}

The diagnosis and management of Laryngopharyngeal Reflux is tough in clinical practice since the symptoms are loosely related to Gastroesophageal Reflux Disease. Specific tools are thus needed which can aid in the assessment of improvement or deterioration of the patient after the treatment modality is administered. One of those tools include the Reflux Symptom Index which is by far the easiest to use because of its noninvasive and time-saving nature and cost effectiveness. In our study, we found that the RSI improved by about $49 \%$ after 3 months and $58 \%$ after 6 months. It can thus 
be concluded that the RSI is a relevant tool to assess patients with LPR in our population.

\section{References}

1. Vaezi MF, Hicks DM, Abelson TI, Richter JE. Laryngeal signs and symptoms and gastroesophageal reflux disease (GERD): a critical assessment of cause and effect association. Clin Gastroenterol Hepatol. 2003 Sep; 1(5): 333-44

2. Patel DA, Blanco M, Vaezi MF. Laryngopharyngeal Reflux and Functional Laryngeal Disorder: Perspective and Common Practice of the General Gastroenterologist. Gastroenterology \& Hepatology. 2018 Sep; 14 (9): 512-20

3. Ahuja V, Yencha MW, Lassen LF. Head and neck manifestations of gastroesophageal reflux disease. Am Fam Physician. 1999; 60(3): 873-80, 885-6

4. Koufman JA. The otolaryngologic manifestations of gastroesophageal reflux disease (GERD): a clinical investigation of 225 patients using ambulatory 24-hour $\mathrm{pH}$ monitoring and an experimental investigation of the role of acid and pepsin in the development of laryngeal injury. Laryngoscope 1991;101(4 pt2) (suppl 53):1-78

5. Merati AL, Lim HJ, Ulualp SO, Toohill RJ. Meta-analysis of upper probe measurements in normal subjects and patients with laryngopharyngeal reflux. Ann OtolRhinolLaryngol. 2005; 114(3): 177-8

6. Shaw MJ, Talley NJ, Beebe TJ, et al. Initial validation of a diagnostic questionnaire for gastroesophageal reflux disease. Am J Gastroenterol. 2001; 96: 52-7

7. Peter C. Belafsky, Gregory N. Postma, James A. Koufman. Validity and Reliability of the Reflux Symptom Index (RSI). Journal of Voice; 16(2):274-7

8. Belafsky PC, Postma GN, Koufman JA. Validity and Reliability of the Reflux Finding Score. Laryngoscope. 2001 Aug; 111(8): $1313-7$
9. Koufman JA. Laryngopharyngeal Reflux is different from classic Gastroesophageal Reflux Disease. Ear, Nose, Throat Journal 2002; 81(9 Suppl 2):7-9

10. Belafsky PC, Postma GN, Koufman JA. Laryngopharyngeal reflux symptoms improve before changes in physical findings. Laryngoscope 2001; 111(6): 979-81

11. Farrokhi F, Vaezi MF. Laryngeal disorders in patients with gastroesophageal reflux disease. Minerva Gastroenterol Dietol. 2007; 53(2): 181-7

12. Pearson J, Parikh S, Orlando R, Johnston N, et al. Review article: reflux and its consequences - the laryngeal, pulmonary and oesophageal manifestations. In: Conference held in conjunction with the 9th international symposium on human pepsin (ISHP) Kingston-upon-Hull, UK, 21-23 April 2010, Aliment Pharmacol Ther. 33 (Suppl. 1): 1-71

13. Kirti YK. Reflux Finding Score (RFS) a Quantitative Guide for Diagnosis and Treatment of Laryngopharyngeal Reflux. Indian J Otolaryngol Head Neck Surg. 2018; 70(3): 362-5

14. Abou-Ismail A, Vaezi MF. Evaluation of patients with suspected laryngopharyngeal reflux: a practical approach. Curr Gastroenterol, Rep. 2011; 13(3):213-8

15. Keshavamurthy VB, Sadashivaooa YB, Harsham NK. Laryngopharyngeal Reflux: Inter-rater Reliability of Reflux Finding Score in Clinical Practice. Bengal Journal of Otolaryngology and Head Neck Surgery 2019; 27(3):186-92

16. Salihefendic N, Zildzic M, Cabric E. Laryngopharyngeal Reflux Disease - LPRD. Med Arch. 2017 Jun;71(3):215-218. doi: 10.5455/medarh.2017.71.215-8

17. Mesallam TA, Stemple JC, Sobeih TM, Elluru RG. Reflux Symptom Index versus Reflux Finding Score. Ann OtolRhinolLaryngol. 2007; 116(6):436-40

18. Nunes HS, Pinto JA, Zavanela AR, Cavallini AF, et al. Comparison between the Reflux Finding Score and the Reflux Symptom Index in the Practice of Otorhinolaryngology. Int Arch Otorhinolaryngol. 2016; 20(3):218-21. doi: 10.1055/s0036-1579557. 